\title{
Clique percolation in random networks
}

\author{
Imre Derényi, ${ }^{1}$ Gergely Palla, ${ }^{2}$ and Tamás Vicsek ${ }^{1,2}$ \\ ${ }^{1}$ Department of Biological Physics, Eötvös University, Pázmány P. stny. 1A, H-1117 Budapest, Hungary \\ ${ }^{2}$ Biological Physics Research Group of HAS, Pázmány P. stny. 1A, H-1117 Budapest, Hungary \\ (submitted to Phys. Rev. Lett. 10 November 2004; accepted for publication 20 April 2005)
}

\begin{abstract}
The notion of $k$-clique percolation in random graphs is introduced, where $k$ is the size of the complete subgraphs whose large scale organizations are analytically and numerically investigated. For the Erdős-Rényi graph of $N$ vertices we obtain that the percolation transition of $k$-cliques takes place when the probability of two vertices being connected by an edge reaches the threshold $p_{\mathrm{c}}(k)=[(k-1) N]^{-1 /(k-1)}$. At the transition point the scaling of the giant component with $N$ is highly non-trivial and depends on $k$. We discuss why clique percolation is a novel and efficient approach to the identification of overlapping communities in large real networks.
\end{abstract}

PACS numbers: 02.10.Ox, 89.75.Hc, 05.70.Fh, 64.60.-i

There has been a quickly growing interest in networks, since they can represent the structure of a wide class of complex systems occurring from the level of cells to society. Data obtained on real networks show that the corresponding graphs exhibit unexpected non-trivial properties, e.g., anomalous degree distributions, diameter, spreading phenomena, clustering coefficient, and correlations [1, 2, 3, 4, 5]. Very recently great attention has been paid to the local structural units of networks. Small and well defined subgraphs have been introduced as "motifs" [6]. Their distribution and clustering properties [6, 7, 8] can be used to interpret global features as well. Somewhat larger units, made up of vertices that are more densely connected to each other than to the rest of the network, are often referred to as communities [9, 10, 11, 12, 13, 14, 15, 16], and have been considered to be the essential structural units of real networks. They have no obvious definition, and most of the recent methods for their identification rely on dividing the network into smaller pieces. The biggest drawback of these methods is that they do not allow for overlapping communities, although overlaps are generally assumed to be crucial features of communities. In this Letter we lay down the fundamentals of a kind of percolation phenomenon on graphs, which can also be used as an effective and deterministic method for uniquely identifying overlapping communities in large real networks [17].

Meanwhile, the various aspects of the classical ErdôsRényi (ER) uncorrelated random graph [18] remain still of great interest since such a graph can serve both as a test bed for checking all sorts of new ideas concerning complex networks in general, and as a prototype to which all other random graphs can be compared. Perhaps the most conspicuous early result on the ER graphs was related to the percolation transition taking place at $p=p_{\mathrm{c}} \equiv 1 / N$, where $p$ is the probability that two vertices are connected by an edge and $N$ is the total number of vertices in the graph. The appearance of a giant component, which is also referred to as the percolating component, results in a dramatic change in the overall topological features of the graph and has been in the center of interest for other networks as well.

In this Letter we address the general question of subgraph percolation in the ER model. We obtain analytic and simulation results related to the appearance of a giant component made of complete subgraphs of $k$ vertices ( $k$-cliques). In particular, we provide an analytic expression for the threshold probability at which the percolation transition of $k$-cliques takes place. The transition is continuous, characterized by non-universal critical exponents, which depend on both $k$ and the way the size of the giant component is measured. Our analytic calculations are in full agreement with the corresponding numerical simulations.

Before we proceed to calculate the threshold and the exponents we need to outline some basic definitions. $k$-cliques, the central objects of our investigation, are defined as complete (fully connected) subgraphs of $k$ vertices [19]. As an illustration, in Fig.1 1all the 3-cliques (triangles) are emphasized with either black or dark gray edges. We also introduce a few new notions specific to our problem. (i) $k$-clique adjacency: two $k$-cliques are adjacent if they share $k-1$ vertices, i.e., if they
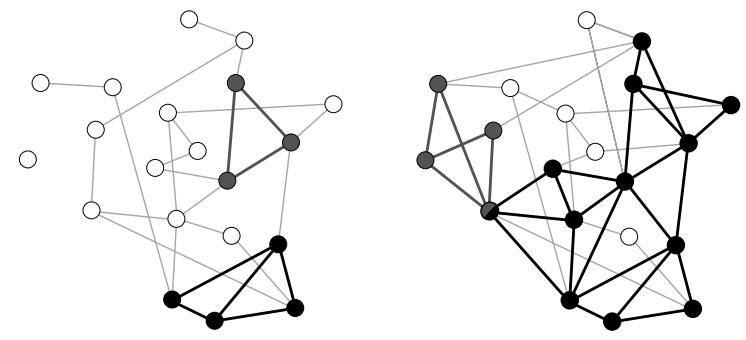

FIG. 1: Sketches of two ER graphs of $N=20$ vertices and with edge probabilities $p=0.13$ (left one) and $p=0.22$ (right one, generated by adding more random edges to the left one). In both cases all the edges belong to a "giant" connected component, because the edge probabilities are much larger than the threshold $\left(p_{\mathrm{c}} \equiv 1 / N=0.05\right)$ for the classical ER percolation transition. However, in the left one $p$ is below the 3 -clique (triangle) percolation threshold, $p_{\mathrm{c}}(3) \approx 0.16$, calculated from Eq. 1, therefore, only two small 3-clique percolation clusters (distinguished by black and dark gray edges) can be observed. In the right graph, on the other hand, $p$ is above this threshold and, as a consequence, most 3-cliques accumulate in a "giant" 3 -clique percolation cluster (black edges). This graph also exhibits an overlap (half black, half dark gray vertex) between two 3-clique percolation clusters (black and dark gray). 
differ only in a single vertex. (ii) $k$-clique chain: a subgraph, which is the union of a sequence of adjacent $k$-cliques. (iii) $k$ clique connectedness: two $k$-cliques are $k$-clique-connected if they are parts of a $k$-clique chain. (iv) $k$-clique percolation cluster (or component): it is a maximal $k$-clique-connected subgraph, i.e., it is the union of all $k$-cliques that are $k$-cliqueconnected to a particular $k$-clique. This is illustrated in Fig. 1] where both graphs contain two 3-clique percolation clusters, the smaller ones in dark gray and the larger ones in black. We note that these objects can be considered as interesting specific cases of the general graph theoretic objects defined in Refs. [20] and [21] in very different contexts.

A $k$-clique percolation cluster is very much like a regular (edge) percolation cluster in the $k$-clique adjacency graph, where the vertices represent the $k$-cliques of the original graph, and there is an edge between two vertices if the corresponding $k$-cliques are adjacent. Moving a particle from one vertex of this adjacency graph to another one along an edge is equivalent to rolling a $k$-clique template from one $k$-clique of the original graph to an adjacent one. A $k$-clique template can be thought of as an object that is isomorphic to a complete graph of $k$ vertices. Such a template can be placed onto any $k$-clique of the original graph, and rolled to an adjacent $k$-clique by relocating one of its vertices and keeping its other $k-1$ vertices fixed. Thus, the $k$-clique percolation clusters of a graph are all those subgraphs that can be fully explored but cannot be left by rolling a $k$-clique template in them.

Now, we present a general result for the threshold probability (critical point) of $k$-clique percolation using heuristic arguments. We find that a giant $k$-clique component appears in an ER graph (as illustrated for $k=3$ in Fig. 11) at $p=p_{\mathrm{c}}(k)$, where

$$
p_{\mathrm{c}}(k)=\frac{1}{[(k-1) N]^{\frac{1}{k-1}}} .
$$

Obviously, for $k=2$ this result agrees with the known percolation threshold $\left(p_{\mathrm{c}}=1 / N\right)$ for ER graphs, because 2-clique connectedness is equivalent to regular (edge) connectedness. Expression (1) can be obtained by requiring that after rolling a $k$-clique template from a $k$-clique to an adjacent one (by relocating one of its vertices), the expectation value of the number of adjacent $k$-cliques, where the template can roll further (by relocating another of its vertices), be equal to 1 at the percolation threshold. The intuitive argument behind this criterion is that a smaller expectation value would result in premature $k$-clique percolation clusters, because starting from any $k$-clique the rolling would quickly come to a halt and, as a consequence, the size of the clusters would decay exponentially. A larger expectation value, on the other hand, would allow an infinite series of bifurcations for the rolling, ensuring that a giant cluster is present in the system. The above expectation value can be estimated as $(k-1)(N-k-1) p^{k-1}$, where the first term $(k-1)$ counts the number of vertices of the template that can be selected for the next relocation, the second term $(N-k-1)$ counts the number of potential destinations for this relocation, out of which only the fraction $p^{k-1}$ is acceptable, because each of the new $k-1$ edges (associated with the relocation) must exist in order to obtain a new $k$-clique. For large $N$, our criterion can thus be written as $(k-1) N p_{\mathrm{c}}^{k-1}=1$, from which we get expression (1) for the threshold probability. The above heuristic approach is similar in spirit to the one used in Ref. [22] in the context of standard percolation on networks.

It is important to point out that this result can be made stronger by a more detailed derivation which we shall present elsewhere due to space limitations. In short, starting from the distribution of the number of $k$-cliques adjacent to a randomly selected one, and applying the so-called generating function formalism [23], one can derive the generating function of the distribution of the number of $k$-cliques that can be visited from a randomly selected one. This function diverges as $p$ approaches $p_{\mathrm{c}}(k)$ from below, signaling the threshold for percolation. Furthermore, our result for $p_{\mathrm{c}}(k)$ is also in perfect agreement with the numerical simulations (see below).

There are two plausible choices to measure the size of the largest $k$-clique percolation cluster. The most natural one, which we denote by $N^{*}$, is the number of vertices belonging to this cluster. We can also define an order parameter associated with this choice as the relative size of that cluster:

$$
\Phi=N^{*} / N
$$

The other choice is the number $\mathcal{N}^{*}$ of $k$-cliques of the largest $k$-clique percolation cluster (or equivalently, the number of vertices of the largest component in the $k$-clique adjacency graph). The associated order parameter is again the relative size of this cluster:

$$
\Psi=\mathcal{N}^{*} / \mathcal{N}
$$

where $\mathcal{N}$ denotes the total number of $k$-cliques in the graph (or the total number of vertices in the adjacency graph). $\mathcal{N}$ can be estimated as

$$
\mathcal{N} \approx\left(\begin{array}{l}
N \\
k
\end{array}\right) p^{k(k-1) / 2} \approx \frac{N^{k}}{k !} p^{k(k-1) / 2},
$$

because $k$ different vertices can be selected in $\left(\begin{array}{l}N \\ k\end{array}\right)$ different ways, and any such selection makes a $k$-clique only if all the $k(k-1) / 2$ edges between these $k$ vertices exist, each with probability $p$. Note that the classical ER percolation is equivalent to our $k=2$ case, and the ER order parameter (relative number of edges) is identical to $\Psi$. Also note that in general the size of the largest cluster could be measured as the number of its $l$-cliques, $\mathcal{N}_{(l)}^{*}$, for $1 \leq l \leq k$. However, for simplicity we restrict ourselves to the two limiting cases $\left(N^{*} \equiv \mathcal{N}_{(1)}^{*}\right.$ and $\left.\mathcal{N}^{*} \equiv \mathcal{N}_{(k)}^{*}\right)$ defined above.

Our computer simulations indicate that the two order parameters behave differently near the threshold probability. To illustrate this, in Figs. $2 \mathrm{k}$ and $3 \mathrm{k}$ we plotted $\Phi$ and $\Psi$, respectively, as a function of $p / p_{\mathrm{c}}(k)$ for $k=4$ and for various system sizes $(N)$, averaged over several runs.

The order parameter $\Phi$ for $k \geq 3$ converges to a step function as $N \rightarrow \infty$. The fact that the step is located at 

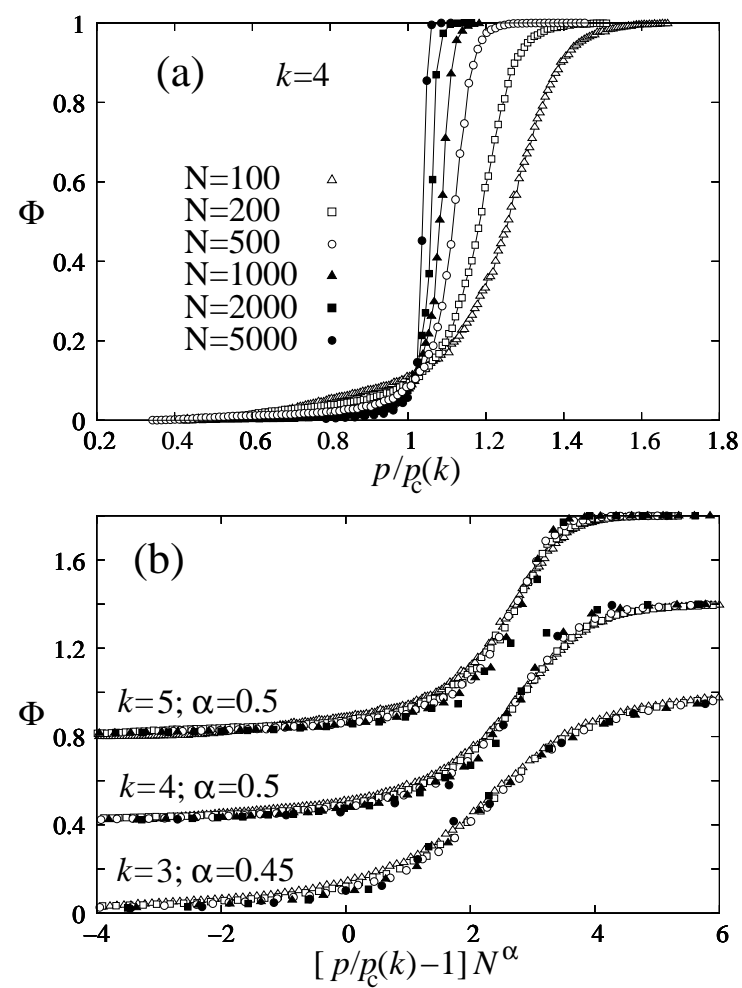

FIG. 2: Simulation results for the order parameter $\Phi$ averaged over several runs (the statistical error is smaller than the symbol size). (a) The convergence of $\Phi$ as a function of $p / p_{\mathrm{c}}(k)$ to a step function in the $N \rightarrow \infty$ limit is illustrated for $k=4$. (b) The width of the steps follows a power law, $\sim N^{-\alpha}$, as the steps collapse onto a single curve if we stretch them out by $N^{\alpha}$ horizontally. The data for $k=4$ and 5 are shifted upward by 0.4 and 0.8 , respectively, for clarity.

$p / p_{\mathrm{c}}(k)=1$ is actually the numerical proof of the validity of our theoretical prediction (1) for $p_{\mathrm{c}}(k)$. The width of the steps follows a power law, $\sim N^{-\alpha}$, with some exponent $\alpha$. Plotting $\Phi$ as a function of $\left[p / p_{\mathrm{c}}(k)-1\right] N^{\alpha}$, i.e., stretching out the horizontal scale by $N^{\alpha}$, the data collapse onto a single curve. This is shown for $k=3,4$, and 5 in Fig. $2 \mathrm{~b}$. The exponent $\alpha$ seems to be around 0.5 for $k \geq 3$. Although for $k=3$ a slight deviation form $\alpha=0.5$ has been obtained, we cannot distinguish that from a possible logarithmic correction.

The order parameter $\Psi$ for $k \geq 2$, on the other hand, similarly to the classical ER transition, converges to a limit function, which is 0 for $p / p_{\mathrm{c}}(k)<1$ and grows continuously from 0 to 1 if we increase $p / p_{\mathrm{c}}(k)$ from 1 to $\infty$.

One of the most fundamental results in random graph theory concerns the behavior of the largest component at the percolation threshold, where it becomes a giant (infinitely large) component in the $N \rightarrow \infty$ limit. Erdős and Rényi showed [18] that for the random graphs they introduced, the size of the largest component $\mathcal{N}^{*}$ (measured as the number of its edges) at $p=p_{\mathrm{c}} \equiv 1 / N$ diverges with the system size as $N^{2 / 3}$, or equivalently, the order parameter $\Psi$ scales as $N^{-1 / 3}$. Since the giant component at the threshold has a tree-like structure, its number of vertices, $N^{*}$, also diverges as $N^{2 / 3}$. We shall show that similar scaling behavior can be obtained for
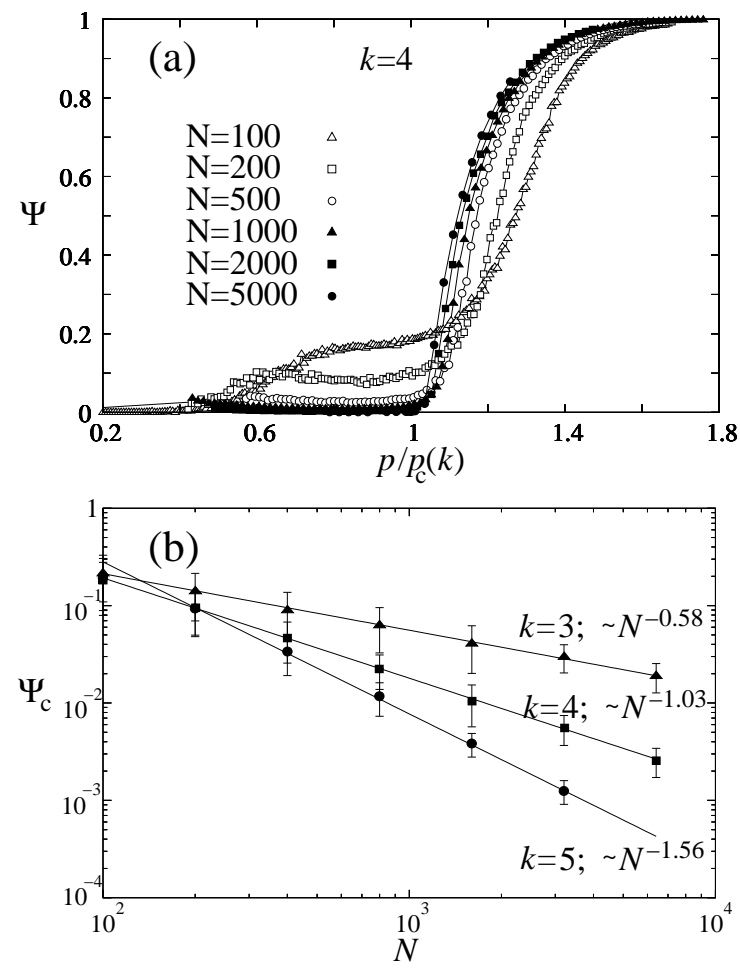

FIG. 3: The order parameter $\Psi$ for the same simulations as in Fig. 2 (a) As illustrated for $k=4, \Psi$ as a function of $p / p_{\mathrm{c}}(k)$ converges to a limit function (which is 0 for $p / p_{\mathrm{c}}(k)<1$ and grows continuously to 1 above $p / p_{\mathrm{c}}(k)=1$ ) in the $N \rightarrow \infty$ limit. (b) The order parameter at the threshold, $\Psi_{\mathrm{c}}$, scales as some negative power of $N$, in good agreement with expression [6.

$k$-clique percolation at the threshold probability $p_{\mathrm{c}}(k)$.

If we assume, that the $k$-clique adjacency graph is like an ER graph [24], then at the threshold the size of its giant component $\mathcal{N}_{\mathrm{c}}^{*}$ scales as $\mathcal{N}_{\mathrm{c}}^{2 / 3}$. The subscript "c" throughout this Letter indicates that the system is at the percolation threshold (or critical point). Plugging $p=p_{\mathrm{c}}$ from Expression (1) into Eq. (4) and omitting the $N$-independent factors we get the scaling

$$
\mathcal{N}_{\mathrm{c}} \sim N^{k / 2}
$$

for the total number of $k$-cliques. Thus, the size of the giant component $\mathcal{N}_{\mathrm{c}}^{*}$ is expected to scale as $\mathcal{N}_{\mathrm{c}}^{2 / 3} \sim N^{k / 3}$ and the order parameter $\Psi_{\mathrm{c}}$ as $\mathcal{N}_{\mathrm{c}}^{2 / 3} / \mathcal{N}_{\mathrm{c}} \sim N^{-k / 6}$.

This is valid, however, only if $k \leq 3$. The reason for the breakdown of the above scaling is that for $k>3$ it predicts that the number of $k$-cliques of the giant $k$-clique percolation cluster, i.e., the number of vertices of the giant component in the $k$-clique adjacency graph, $\mathcal{N}_{\mathrm{c}}^{2 / 3} \sim N^{k / 3}$, grows faster than $N$. On the other hand, in analogy with the structure of the giant component of the classical ER problem, we expect that the giant component in the adjacency graph also has a tree-like structure at the threshold, with very few loops. As a consequence, almost every vertex of the adjacency graph corresponds to a vertex of the original graph. Thus, in the adjacency graph the giant component should not grow faster 
than $N$ at the threshold. Therefore, for $k>3$ we expect that $\mathcal{N}_{\mathrm{c}}^{*} \sim N$, and using Eq. (5), $\Psi_{\mathrm{c}}=\mathcal{N}_{\mathrm{c}}^{*} / \mathcal{N}_{\mathrm{c}} \sim N^{1-k / 2}$. In summary:

$$
\Psi_{\mathrm{c}} \sim\left\{\begin{array}{ll}
N^{-k / 6} & \text { for } k \leq 3 \\
N^{1-k / 2} & \text { for } k \geq 3
\end{array} .\right.
$$

We have also determined the scaling of $\Psi_{\mathrm{c}}$ at $p_{\mathrm{c}}$ as a function of $N$ numerically, and the results are in good agreement with the above heuristic arguments, as shown in Fig. 3 b.

Finally, we discuss the relevance of our approach to community finding $[9,10,11,12,13,14,15]$. If we start rolling a $k$-clique template from a highly connected part of a network we can proceed and find all vertices that can be reached from the initial $k$-clique. Such a $k$-clique percolation cluster can be identified as a community, because of the many (at least $k-1$ ) links of any of its vertices to the other vertices of this cluster. The links are organized into complete subgraphs ( $k$-cliques), which is also a characteristics of most communities (just think of human relations). With different values of $k$ we can identify communities of different strength (or cohesiveness). Our $k$-clique percolation clusters also satisfy a number of basic requirements (local; density based; not too restrictive; have no cut-node; allow overlaps) that are expected from a community definition, but are not satisfied simultaneously by any other existing definition in the literature [20, 25]. Although using $k$-cliques might seem to be a very strict constraint on the community definition, we note that relaxing this constraint (e.g., by allowing incomplete $k$-cliques) is practically equivalent to lowering the value of $k$.

The sharp percolation transition (step in $\Phi$ ) of the ER graphs provides the theoretical basis for the applicability of our community definition to real networks. This is because if the network was completely random, only very few and small clusters would be expected for any $k$ at which the network is below the transition point. However, if large clusters do appear, they must correspond to locally dense structures, i.e., real communities. Moreover, since these communities are locally above the percolation threshold, their identification is immune to random removal of edges as long as their edge density remains above the threshold.

The most important aspect of such a method is that naturally, a single vertex can be part of several communities [17], as illustrated in Fig. 1 (right) by the half black, half dark gray vertex. In terms of a person, he/she can belong to a number of groups (of highly connected people) in such a way that no two groups share a $(k-1)$-clique (there are no $k-1$ people in any two groups who would all know each other and, therefore, would allow a $k$-clique template to roll through). Thus, each vertex can belong to a number of individually identifiable communities and, in turn, each community can have a large number of contacts with other communities, just as it happens in most realistic situations (see, e.g., Ref. [26]). This is very much in contrast with the divisive and agglomerative methods, which force each vertex to belong to only one community and be separated from the others, leading to the loss of many of the communities of the network.

The approach presented in this Letter allows a number of generalizations (e.g., $k$-cliques connected through $(k-l)$ cliques, $k$-cliques with weighted edges, etc.) and opens new directions in the study of network structures made of highly interconnected parts including communities overlapping in various non-trivial ways. As an important biological example, we have successfully applied our method to the identification of protein communities in the protein-protein interaction network of yeast, which has allowed us to make predictions for the yet unknown function of some proteins [17].

This work has been supported in part by the Hungarian Science Foundation (OTKA), grant Nos. F047203 and T034995.

[1] D. J. Watts and S. H. Strogatz, Nature 393, 440 (1998).

[2] A.-L. Barabási and R. Albert, Science 286, 509 (1999).

[3] R. Albert and A.-L. Barabási, Rev. Mod. Phys. 74, 47 (2002).

[4] J. F. F. Mendes and S. N. Dorogovtsev, Evolution of Networks: From Biological Nets to the Internet and WWW (Oxford University Press, Oxford, 2003).

[5] A. Barrat, M. Barthelemy, and A. Vespignani, Phys. Rev. Lett. 92, 228701 (2004).

[6] R. Milo, S. Shen-Orr, S. Itzkovitz, N. Kashtan, D. Chklovskii, and U. Alon, Science 298, 824, (2002).

[7] A. Vazquez, R. Dobrin, D. Sergi, J.-P. Eckmann, Z. Oltvai and A.-L. Barabási, cond-mat/0408431

[8] J.-P. Onnela, J. Saramäki, J. Kertész, and K. Kaski, cond-mat/0408629

[9] M. Blatt, S. Wiseman, and E. Domany, Phys. Rev. Lett. 76, 3251 (1996).

[10] M. Girvan and M. E. J. Newman, Proc. Natl. Acad. Sci. USA 99, 7821 (2002).

[11] H. Zhou, Phys. Rev. E 67, 061901 (2003).

[12] M. E. J. Newman, Phys. Rev. E 69, 066133 (2004).

[13] F. Radicchi, C. Castellano, F. Cecconi, V. Loreto, and D. Parisi, Proc. Natl. Acad. Sci. USA 101, 2658 (2004).

[14] L. Donetti and M. A. Muñoz, cond-mat/0404652

[15] D. M. Wilkinson and B. A. Huberman, Proc. Natl. Acad. Sci. USA 101, 5241 (2004).

[16] J. Reichardt and S. Bornholdt, Phys. Rev. Lett. 93, 218701 (2004).

[17] G. Palla, I. Derényi, I. Farkas, and T. Vicsek, (to appear in Nature).

[18] P. Erdős and A. Rényi, Publ. Math. Inst. Hung. Acad. Sci. 5, 17 (1960).

[19] B. Bollobás, Random graphs, 2nd ed. (Cambridge University Press, Cambridge, 2001).

[20] M. G. Everett and S. P. Borgatti, Connections 21, 49 (1998).

[21] V. Batagelj and M. Zaversnik, arXiv:cs.DS/0308011 (2003).

[22] R. Cohen, K. Erez, D. ben-Avraham, and S. Havlin, Phys. Rev. Lett. 85, 4626 (2000).

[23] M. E. J. Newman, S. H. Strogatz, and D. J. Watts, Phys. Rev. E 64, 026118 (2001).

[24] This assumption is an approximation since the adjacency graph is weakly assortative.

[25] S. Kosub, Network Analysis, LNCS 3418, edited by U. Brandes and T. Erlebach (Springer, Berlin, 2005) pp. 112-142.

[26] J. Scott, Social Network Analysis: A Handbook, 2nd ed. (Sage Publications, London, 2000). 